\title{
The Most Logical Cancer Therapy Concept in The World?
}

\author{
Manfred Doepp* \\ Holistic Center Head, Abtwil 9030, Switzerland \\ *Corresponding author: Manfred Doepp, Holistic Center, 13 Haupt St, Abtwil 9030, Switzerland. \\ To Cite This Article: Manfred Doepp. The Most Logical Cancer Therapy Concept in The World?. Am J Biomed Sci \& Res. 2021 - 13(6). AJBSR. \\ MS.ID.001934. DOI: 10.34297/AJBSR.2021.13.001934.
}

Received: 制 July 22, 2021; Published: 海 August 17, 2021

\section{Opinion}

If one looks at the statistics on cancer incidence worldwide and at the cure rates - at least over 5 years - you will see that we are further away than ever from beating cancer (as President Nixon announced at the time). There are cancers where cure rates are close to zero, and there are others whose incidence is exploding. The predominant therapy still consists of the old trias: scalpel, chemotherapy and radiation. In the field of chemotherapy, new ways and means are constantly being brought onto the market, such as immunotherapy. It is very expensive and has only slightly increased cure rates. One problem is regularly that the untargeted chemotherapy kills the sensitive cells but does not reach the resistant cells. These can then multiply at will.

The crux of the matter is that although oncologists repeatedly promise personalisation or individualisation instead of regimens, this hardly ever happens in routine. And it is only these conditions, however, that can provide a leap forward: tumour-specific and individualised chemotherapy. A laboratory method exists for this purpose: the "liquid biopsy". It manages more than 3 million mentions on Google and is described as globally recognised in Wikipedia. We have been performing it on every cancer patient for more than a year (www.rgcc-international.com). To do this, $40 \mathrm{ml}$ of blood is sent to a special laboratory in Greece. There, the cancer cells are selected and multiplied many times. With their help, one can now recognise the type of cancer, one can test all existing chemotherapeutic agents for their effectiveness on the cancer cells and also test about 60 potentially cytotoxically effective natural remedies.

\section{The RGCC laboratory writes: With our tests we pursue three goals}

A. Potential early detection of an undiagnosed cancer.

B. Monitoring of already diagnosed cancers.

C. Individualised advice on the use of chemotherapeutic agents and natural substances from which individual patients can benefit." The result lists are rankings of effectiveness in killing cancer cells. Individual therapy can now be put together from the best-performing chemical and natural agents. Of the chemical agents, for example, the ancient cisplatin has proven effective, and of the natural agents, Angiostop $₫$, made from sea cucumber. But Artemisia annua, the annual mugwort, also proves its abilities, as does high-dose intravenous vitamin $\mathrm{C}$.

We have made the experience that individualisation allows a reduction of the dosage in chemotherapy, to about $50 \%$, the socalled "low-dose chemo". It has considerably fewer side effects than high-dose chemo. As a third pillar of therapy, we use intracellular enzymes from the company Citozeatec: www.citozeatecsrl.ch. They are used by means of tumour-specific intake protocols. The enzymes do not have a cytotoxic effect but convert the lactate metabolism typical of cancer and back into oxygen metabolism. The cancer cells can thus be "resocialised". Enzymes have proven their abilities in a cancer study at the Tor Vergata University in Rome: https://pubmed.ncbi.nlm.nih.gov/32034492/. Since the enzymes are nature-identical, they can be used via all existing access routes: oral, nasal, inhalation, anal, vaginal, subcutaneous, intravenous. 
The fourth pillar is SOT: Supportive Oligonucleotide Technique. https://biomedical-center.de/sot-therapie/.

SOT has the ability to induce apoptosis (cell death) in CTCs, CSCs and primary tumour cells. CTCs are identified from a blood sample, then a small molecule so-called microRNA is developed that precisely matches a "lock" part of the cancer cell that controls vital cell functions. When injected intravenously, SOT spreads throughout the body, including across the blood-brain barrier, embedding itself in the cancer and disrupting the cancer's or pathogen's ability to replicate. SOT has the ability to prevent destruction and works around the clock to fight the cancer for up to 6 months.

The risks of SOT infusion are primarily related to the sudden death of the cancer cells. If there is a large tumour burden, cell death can produce a large amount of dead cell debris leading to tumour lysis syndrome. To minimise the risk in this situation, a lower dose of SOT is given or a weaker SOT is done. Prior to SOT treatment, a recent PET/CT scan is recommended to assess the current tumour burden. Prior to SOT therapy, a blood draw to count circulating tumour cells using RGCC (oncocount, oncotrace, oncotrail, etc.) is required, and ideally this count is repeated at each SOT treatment to monitor CTC counts and/or account for cancer cell mutations.

\section{Our Cancer Concept is Thus as Follows}

1. Liquid biopsy (RGCC) with identification of individually effective chemotherapeutic agents. The three best are used intravenously as low-dose chemo.
2. Application of the six most effective natural remedies, orally or - if possible - intravenously.

3. Parallel implementation of enzyme therapy according to the protocol of the cancer type, via as many access routes as possible.

4. Application of the SOT (Supportive Oligonucleotide Technique).

5. As we as a small center are not in a position to carry out the chemotherapy ourselves by infusion, this is done in a cooperative clinic. The laboratory in Greece is also constantly testing new agents that promise success. For example, we had a tincture tested that contains hydrogen peroxide. It proved to be effective against viruses, bacteria and - to our astonishment - also against cancer cells. The same is true for colchicine.

6. So far, we have good experience with this concept. We think it is the most logical one that can be applied in real life at present. 